\title{
Research Progress of Graphene and Its Derivatives in Cancer diagnosis and Therapy
}

\author{
ZhenBang Sun ${ }^{1, \mathrm{a}}$, XiuHong Chen², LiHui Zhao ${ }^{3 *}$ \\ ${ }^{1}$ College of Life Science and Technology/Chang Chun University of Technology /Jilin China, 282977643@qq.com \\ ${ }^{2}$ College of Life Science and Technology /Chang Chun University of Technology /Jilin China, 1252042806@qq.com \\ ${ }^{3}$ College of Life Science and Technology /Chang Chun University of Technology/Jilin China,
}

\begin{abstract}
Because of its high incidence rate and difficult to treature, tumor has long been a hot topic in modern medical research. The traditional cancer therapy is still based on surgery, radiotherapy and chemotherapy.Although the medical technology has been optimized and innovated, it is constantly improved and tends to be mature. However, radiotherapy and chemotherapy have great side effects on human body, and the damage to normal tissues can not be underestimated, which brings great pain to patients. The development of new methods of tumor treatment has become a hot spot in the field of anti-tumor. In this paper, the diagnostic and therapeutic methods of graphene and its derivatives were reviewed.
\end{abstract}

\section{Introduction}

Nano materials refer to the materials whose size is between $1 \sim 100 \mathrm{~nm}$ or the materials with this structure as the basic unit. Nanomaterials have been playing an important role in production and life due to their small size. In recent years, the application of biomedical materials and tumor treatment in biomedical field has been developing rapidly, such as drug delivery carrier, diagnosis and treatment probe, photothermal imaging and photothermal therapy. Graphene is one of the most popular nano materials. Due to its unique hexagonal structure, graphene has obtained other good physical and chemical properties except for the small size of nano materials. It has unique electronic structure and good conductive effect, has the affinity of non-metallic particles to organism, good photo thermal conversion ability and easy modification ability. These characteristics make it widely used in the field of biomedicine. It can be used as a carrier of targeted drugs, as well as anti-tumor.

\section{2 structure and physical and chemical properties of graphene}

In 2004, British scientists Andre Geim and Konstantin Novoselov obtained graphite flakes from highly oriented pyrolytic graphite (HOPG) by micromechanical stripping method. It was found that HOPG is a two-dimensional carbon nanomaterial with a honeycomb lattice and hexagonal structure composed of carbon atoms with SP2 hybrid orbitals (Figure 1.) .Its thickness is about $0.35 \mathrm{~nm}$, which is called graphene[1], Graphene oxide (NGO) has good biocompatibility and water solubility, and has no obvious cytotoxicity. GO has many excellent physical and chemical properties,such as special molecular structure, large specific surface area, excellent optical, electrical, mechanical and thermal properties. Its unique twodimensional structure makes it possess the comprehensive properties of graphite and carbon nanotubes at the same time, showing good mechanical properties, photothermal ability and biological affinity, which makes it have great application in biomedical field Prospects [2].

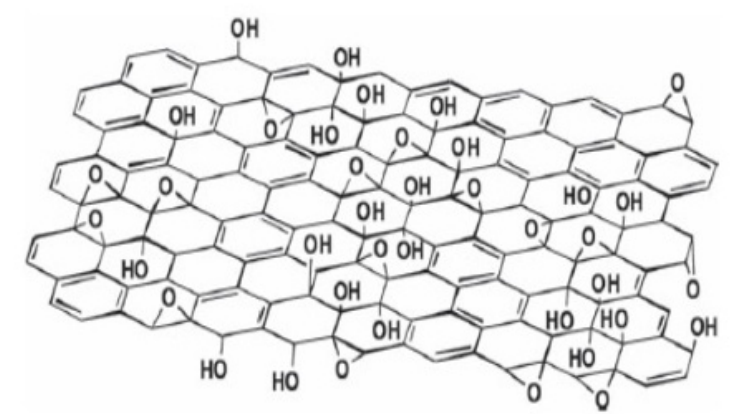

Fig1. The structure of graphene

\section{Research Progress on antitumor effect of graphene and its derivatives}

The properties of drugs have little effect on the distribution of drugs in vivo, which is more dependent on the properties of different drug carriers. A good drug carrier can accurately locate the lesion tissue, release the drug in a controlled manner, and do not damage the normal tissue. Therefore, in order to achieve accurate targeted drug delivery effect, it is necessary to develop more stable, safe and efficient drug carriers. Due to the excellent biological properties of graphene and its derivatives. In recent years, graphene oxide, as a kind of nano carrier, has 
become a hot spot in the research of antitumor drugs. Graphene oxide loaded with antitumor drugs can improve the biological activity of anti-tumor drugs, enhance the practicability, safety and reduce the side effects of antitumor drugs. At the same time, graphene has special physical structure, good mechanical properties, large specific surface area, small volume and other characteristics, which can make graphene loaded antitumor drugs reach the tumor site through enhanced permeability and retention effect (EPR)[3], which can increase the anti-tumor effect and is an ideal drug carrier in the field of biomedical anti-tumor. The two-dimensional nanostructure and its benzene ring structure endow graphene with the ability to be different from other molecules, so that its affinity with organic molecules has been improved, so that it has a great development in the field of biomedicine, especially in the field of anti-tumor. Zhang Yajing et al. Prepared nano graphene oxide (NGO) with graphene as carrier, and prepared ng0-peg-bpei-cddp nano drug delivery system by using polyethylene glycol (PEG) and polyethyleneimine (bpei) modified nano graphene oxide (CDDP) to prepare NGO-PEG-bPEICDDP nano drug delivery system. Through experiments, it was found that the system has good cell targeting effect and anti-tumor effect [4]. Wu Honghui et al. Used doxorubicin (DOX) and topotecan (TPT) to connect with graphene oxide (go) to obtain a drug-loaded targeting system. The system can achieve targeted positioning and sustained-release effect according to the different $\mathrm{pH}$ value of pathological cells and normal cells, and has good killing effect on tumor [5]. In recent years, the latest research on the controlled-release performance and biological safety of graphene oxide and its derivatives as drug carriers also confirmed that graphene oxide has the advantages of passive targeting and a large number of accessible active groups, and is an ideal carrier for the development of nano targeted drug delivery and transportation [6-7].

Graphene oxide and its derivatives as a drug targeted therapy for cancer, another way of thinking is tumor photothermal therapy (PTT). The good photothermal conversion properties of graphene oxide also endow it with excellent application potential in the photothermal treatment of tumor. Graphene oxide enters tumor cells and can specifically raise the temperature of tumor cells when high energy irradiation is used.The normal tissues and cells of the body will not be damaged if the cells are killed. Therefore, Huang Chunzhi et al. Developed an active targeting nano drug delivery system of graphene oxide based on the photothermal properties of graphene and its use as drug carrier. The nanocomposite system can actively target the tumor site sensitive to $\mathrm{pH}$, which greatly reduces the amount of chemical drugs. At the same time, it has the ability of photothermal treatment and is the target of tumor It provides a new idea and method for combined treatment [8]. Graphene, as a photothermal conversion material, reaches the tumor cells. After infrared light irradiation, the fever kills the tumor cells. Li Yong et al. Irradiated tumor tissue injected with nano graphene oxide (NGO) with $805 \mathrm{~nm}$ near-infrared laser. The experiment confirmed that NGO has good photothermal conversion effect, can stimulate the immune effect of macrophages in vivo, induce the body's anti-tumor immune response, and achieve the effect of inhibiting and isolating tumor growth[9]. Small size go thin films show high NIR light absorption and biocompatibility, which can be used as potential materials for photothermal therapy.Transdermal nano go hyaluronic acid (NGO-HA) conjugates can be used to treat melanoma under near-infrared laser irradiation. A series of organic-inorganic complexes (SSGPDs) were synthesized by atom transfer radical polymerization (ATRP) containing disulfide bond initiation sites on go surface, and then modified go with ATRP and (2-diamino) ethyl methacrylate (DMAEMA). SS-GPDs materials can attach and absorb aromatic waterinsoluble drugs, and can effectively kill cancer cells due to the conjugate structure of GO substrate plane [10].

Graphene oxide and its derivatives can be used as carriers of photosensitizers in photodynamic therapy (PDT), which can make up for the shortcoming that photosensitizers can only act on superficial tumors because of the short wavelength. The photothermal / twophoton photodynamic therapy based on graphene oxide has good biocompatibility. Deng Lidong et al. Developed the photothermal / two-photon photodynamic therapy to break the limitation of the physical and chemical properties of photosensitizers, and further confirmed that the photothermal / two-photon photodynamic therapy has obvious inhibitory effect on tumor growth in mice. The new two-photon photodynamic therapy (TPD) uses twophoton absorption (2PA) compounds to absorb the energy of long wavelength light source, and transfer the energy to photosensitizer through fluorescence resonance energy transfer (FRET), and then produce reactive oxygen species such as single line oxygen. In theory, the twophoton photodynamic therapy overcomes the weak penetration of photodynamic therapy [11], and is expected to become a new way to treat cancer in the future.

In recent years, more and more attention has been paid to the immunological effects of go and its derivatives. Due to the important role of macrophages in innate immunity, the effects of go and GO-PEG on morphology and biological function of mouse macrophage RAW264.7 were studied. The results showed that the cytoplasmic vacuoles of RAW264.7 cells were inhibited. After GO treatment, IL-1 $\beta$ and IL-10 were down regulated, IL-6 was up-regulated, and TNF - $\alpha$ was not changed. Further studies have shown that GO can also induce autophagy in a variety of cells, such as mouse macrophage line ANA-1, mouse peritoneal macrophage, mouse fibroblast-NIH3T3. However, different from GO, GO-PEG could not induce autophagy, and the morphology and function of cells did not change significantly before and after treatment, which indicated that the interaction between GO and cells might be reduced or changed by PEG on the surface. Then, the potential mechanisms of autophagy induced by go were systematically studied by various methods, including signal pathway inhibitors, RNA interference techniques, Western blot and flow cytometry. It reveals the mechanism of macrophage autophagy mediated by GO, and provides important theoretical support for further exploring the immunological effects of GO and its derivatives, especially the interaction mechanism between GO and immune cells [12]. 


\section{Application of graphene and its derivatives in tumor diagnosis}

\subsection{Application of graphene in magnetic resonance imaging (MRI)}

Magnetic resonance imaging (MRI) technology is a nondestructive testing method for rapid detection of visceral organs and soft tissues, which plays an important role in the detection and diagnosis of visceral organ diseases, especially tumors[13]. By connecting graphene oxide with magnetic material particles, the behavior of graphene in vivo can be observed by MRI technology. This composite material may have the ability of MRI contrast agent and tumor treatment drug carrier at the same time, so as to realize the magnetic visualization and targeting of drug release [14]. The above-mentioned drug delivery system, which combines graphene with magnetic nanoparticles, is controllable, simple, and capable of enriching drugs in lesion sites. It will have important applications in nuclear magnetic resonance imaging, tumor diagnosis and targeted therapy [15].

Wang Yapei et al. Prepared a nano hybrid material GO / $\mathrm{MnFe}_{2} \mathrm{O}_{4}$ as a new type of MRI contrast agent, imaging studies in tumor mice showed that the composite system will gather in the tumor site after a period of time, so it can be used as cancer diagnosis material. At the same time, $\mathrm{GO} / \mathrm{MnFe}_{2} \mathrm{O}_{4}$ hybrid system can be absorbed by liver, lung and spleen, and can be metabolized from liver and kidney in vitro[16]. The research results of Wang Yapei and others have laid a foundation for the application of graphene in nuclear magnetic resonance imaging. Go / magnetic composite system will also become the mainstream application material in the field of nuclear magnetic resonance imaging and tumor treatment.

\subsection{Application of graphene and its derivatives in tumor detection}

Early tumor symptoms are small, not easy to detect, does not affect people's daily life and is not easy to be detected, so most of the confirmed cases of cancer are found to be in the middle and late stage, which leads to miss the best period of treatment, so the early detection and diagnosis of tumor is very important. Graphene has good modifiable properties and stability, and has great application prospects in tumor detection. For example, Wang Jing et al. Constructed a tumor marker alpha fetoprotein based on graphene oxide, The detection range is $3.5 \mathrm{pg} / \mathrm{mL}-35$ $\mathrm{ng} / \mathrm{mL}$, and the detection limit is up to $106 \mathrm{fg} / \mathrm{mL}$, which provides a new idea and direction for the research of graphene in tumor detection[17]. The 8-nanometer-thick graphene was evenly spread on one side of a prism, and a cell channel was built close to the graphene. In the experiment, a beam of light from one side of the prism, through graphene irradiation on the cell channel, reflected light from the other side of the prism. Because the shape, size and nature of cancer cells are different from those of normal cells, the influence of cancer cells on the polarization of reflected light is quite different from that of normal cells. The experimental personnel can get a waveform by photoelectric conversion. If there are cancer cells in the cell channel, the oscillogram will show obvious wave crest. Field experiments show that even if one of the thousands of normal cells has a lesion, this "light detection" method can accurately identify it. The advantage of this method is that it can detect the flow cell samples quickly. If it is applied in the future, it will greatly advance the time when cancer cells are found, which will help people prevent cancer and provide early intervention and treatment.

\section{Biosafety of graphene and its derivatives}

The biological safety of nanomaterials directly determines their application prospects in biomedical field. Graphene oxide and its derivatives have shown good application prospects in the biomedical field. The extensive application in production and life also makes people worry about their safety, so it also leads to the research on their biological safety. The current research results show that the physical and chemical properties of graphene, such as size, surface physical and chemical properties, can significantly affect its biological effects and toxicity. The cytotoxicity of graphene and its derivatives is size and concentration dependent (the toxicity of go is also related to the degree of oxidation), which usually shows good biocompatibility in a certain concentration range. When the concentration of graphene exceeds a certain limit, it will produce some adverse reactions (such as cell membrane damage, decreased cell activity, etc.); in addition, graphene and go have certain hemolytic activity. Fortunately, the modification of polyethylene glycol, chitosan and protein can effectively reduce the cytotoxicity and hemolysis of graphene materials at high concentrations. Liu Sijin et al. Found that one of the mechanisms of cytotoxicity of GO is: GO can affect the structure and functional proteins in cell membrane, resulting in abnormal structure and physical and chemical properties of cell membrane. Therefore, by modifying GO to change its surface structure, thus changing its physical and chemical properties, the damage of GO on cell membrane can be reduced, and then the in vivo toxicity caused by GO can be reduced [18]. Therefore, we do not have to worry too much about the biosafety and stability of GO. We can change the physical and chemical properties of graphene and its surface by functionalization and detoxification modification, so as to achieve the purpose of more safety.

\section{Expectation}

With its unique structure and unparalleled performance, graphene nanodrug delivery system has become a hot topics in the field of biomedical research, especially in the field of anti-tumor immunotherapy.At the same time, it has great guiding significance and development prospects for the research of cancer with unclear and complex types.In recent years, tumor targeted therapy has made certain progress, and some new tumor targeting drugs have appeared in clinical trials, which have shown good anti- 
tumor ability. However, the research on tumor inhibitors always brings out worrying results, and the typical problem is drug resistance. At the same time, there are still some problems, such as relatively large side effects, insufficient application range, unsatisfactory treatment effect. At present, the main research enthusiasm of current researchers has gradually transferred to the immunotherapy of tumor, and achieved certain results, but also exposed the expansion efficiency of in vitro immune cells, the standardization of preparation, and the lack of randomized controlled studies[19].Among them,the research of immunotherapy combined with other therapies is becoming a new hot topic in cancer treatment research, and graphene nano drug delivery system is an excellent bridge connecting these combined therapy. Graphene nano drug delivery system not only develops in the fields of tumor immune treatment, targeted therapy, photothermal therapy and chemical drug therapy, but also has some development. The combined application of some therapies is brilliant, so as to overcome the shortcomings of these therapies for anti-tumor treatment alone, and further promote the development of anti-tumor treatment in the biomedical field. It is believed that with the further research and development of graphene and its derivatives, it will play an irreplaceable role in cancer treatment, thus promoting the technological revolution in the field of cancer treatment.

\section{Conclusion}

Graphene and its derivatives have the characteristics of good water solution stability, biocompatibility, high fluorescence display efficiency and large specific surface area. Its application value in biomedical field is gradually highlighted. Scholars from various countries have gathered to carry out a lot of frontier research and obtained remarkable results. Graphene and its derivatives have become the most promising biomaterial in biomedical applications. It has high drug loading capacity, and has become a research hotspot in gene delivery, biological imaging, photodynamic therapy, immune enhancement and antibacterial effects.

\section{ACKNOWLEDGMENTS}

Thanks for the support from Harbin baiaokang Biomedical Technology Co., Ltd.

\section{REFERENCES}

1. Novoselov, K.S., Geim, A.K., Morozov,et al (2004) .Electric field effect in atomically thin carbon films [J]. Science, 306: 666-669.

2. Jiang Hong, Wang Chunxiao(2019). Research progress of graphene frontier application at home and abroad [J]. New materials industry, (11): 11-15.

3. Li Li(2019). Construction of biomimetic nano drug delivery system based on composite membrane and its anti-tumor treatment research [D]. Zhengzhou University.
4. Zhang Yajing(2019). Preparation and antitumor effect of graphene nanoparticle drug delivery system [D]. Changchun University of science and technology.

5. Wu Honghui(2015). Controlled release and antitumor application of graphene oxide [D]. Hunan University.

6. Yang $\mathrm{K}$, Zhang $\mathrm{S}$, Zhang G,SunX,et al.(2010).Graphene in Mice:Ultrahigh In Vivo Tumor Uptake and Eficient Photothermal Therapy. Nano letters, 10 (9): 3318-3323.

7. Qian Wenhao, Su Jiansheng(2016). Research status of controlled release performance of functionalized graphene oxide $[\mathrm{J}]$. Journal of Shanghai Second University of technology,2:88-93.

8. Huang Chunzhi(2018). Study on active targeting nano drug delivery system of graphene oxide with synergistic chemotherapy and photothermal therapy [D].Shandong University.

9. Li Yong, Zhou Fu, Chen Wei(2017). Graphene oxide mediated photothermal immunotherapy for metastatic mouse breast tumor $[\mathrm{J}]$. Progress in biochemistry and Biophysics,44 (12): 1095-1102.

10. Chiang. C S, Lin Y J, Lee R, et al.( 2018). Combination of fucoidan-based magnetic nanoparticles and immunomodulators enhances tumour-localized immunotherapy[J]. Nat Nanotechnol, 13: 746-754.

11. Deng Lidong(2017).Preliminary study on photothermal two-photon photodynamic therapy based on graphene oxide as drug carrier [D]. Southwest University.

12. Wang Ting(2015).Interaction between functionalized graphene oxide and macrophages and its mechanism [D]. Suzhou University.

13. Xiaodan Huanga, Xufeng Zhou, Kun Qi an,et al. (2012). A magnetite nanocrystal/gr aphene composite as high performance anode for lithium-ion batteri es[J]. Joumal of Alloys and Compounds,514,76-80.

14. Xuexia Liua,b, Hui Zhua, Xiurong Yang (2011). An amperometric hydrogen peroxide chemical sensor based on graphene-Fe3O4 multilayer films modified ITO el ectrode[J]. Talanta ,87,243-248.

15. Q. Ou, T. Tanaka, M. Mesko, A. Ogino, M. Nagatsu. (2008). Char acteristics of graphene-layer encapsul ated nanoparticl es fabricated using laser ablation method[J]. Diamond \& Related Materials ,17, 664668.

16. Wang Yapei(2013). Preparation of functionalized graphene oxide and its application in drug delivery and magnetic resonance imaging [D]. Shanghai Normal University.

17. Wang Jing(2019). Preparation of polymer / graphene oxide composites and high sensitivity detection of tumor markers [D]. Ludong University.

18. Cheng Xianzhen, Zhou Yi, Jiang Zaixing(2016). Preliminary study on the safety of graphene oxide in rats $[\mathrm{J}]$. Biomedical engineering and clinical, 4:350354.

19. Niu Lingling, Jin Yifeng(2019). Advances in targeted 
therapy and immunotherapy for lung cancer [J]. Journal of Mudanjiang Medical College,40 (01): 9397. 